\title{
Enhanced electrochemical performances of activated carbon (AC)-nickel-metal organic framework (SIFSIX-3-Ni) composite and ion-gel electrolyte based supercapacitor
}

\author{
Kyu Seok Lee ${ }^{1} \cdot$ Ye Ji Seo $^{1} \cdot$ Hyeon Taek Jeong $^{1}$ (D)
}

Received: 30 October 2020 / Revised: 14 December 2020 / Accepted: 15 December 2020 / Published online: 4 January 2021

(c) The Author(s) 2021

\begin{abstract}
In this study, we investigated that the activated carbon (AC)-based supercapacitor and introduced SIFSIX-3-Ni as a porous conducting additive to increase its electrochemical performances of AC/SIFSIX-3-Ni composite-based supercapacitor. The AC/SIFSIX-3-Ni composites are coated onto the aluminum substrate using the doctor blade method and conducted an ion-gel electrolyte to produce a symmetrical supercapacitor. The electrochemical properties of the AC/SIFSIX-3-Ni composite-based supercapacitor are evaluated through cyclic voltammetry (CV), electrochemical impedance spectroscopy (EIS), and galvanostatic charge/discharge tests (GCD). The AC/SIFSIX-3-Ni composite-based supercapacitor showed reasonable capacitive behavior in various electrochemical measurements, including CV, EIS, and GCD. The highest specific capacitance of the AC/SIFSIX-3-Ni composite-based supercapacitor was $129 \mathrm{~F} \mathrm{~g}^{-1}$ at $20 \mathrm{mV} \mathrm{s}^{-1}$.
\end{abstract}

Keywords Supercapacitor $\cdot$ SIFSIX-3-Ni $\cdot$ AC/SIFSIX-3-Ni composite $\cdot$ Ion-gel electrolyte

\section{Introduction}

As the demand for energy to replace fossil fuels has increased, the energy storage devices have begun to attract attention [1,2]. Due to these demands, many studies are still struggling to increase the energy and power density of energy storage devices, including batteries, fuel cells, and supercapacitors(SCs) [3, 4]. SCs are promising devices compared to batteries and fuel cells [5]. SCs have high power density, chemical stability, and cycle life of more than 10,000 cycles [4]. SCs are also desired for various applications, such as electric vehicles, portable devices, and wearable electronics [6]. There are two types of SCs, depending on the charge/discharge mechanism. The first is the electrochemical double-layer capacitor (EDLC), which is worked by the electric double layer that is formed at the electrode/electrolyte surface [7,8]. As the electrode material for EDLC, carbon materials with a large surface area, such

Hyeon Taek Jeong

jht4321@daejin.ac.kr

1 Division of Energy and Environmental Engineering,

Daejin University, 1007 Hoguk Road, Pocheon-si,

Gyeonggi-do 487-711, South Korea as graphene, activated carbon, and carbon nanotubes, are mainly conducted [1,9-12]. The second type is a pseudocapacitor, where electric charges are stored by the faradaic reaction. The pseudocapacitor mostly uses the transition metal oxides, conducting polymer and metal-organic frameworks (MOFs) for the electrode materials [1, 6, 7, 13, 14].

In an effort to improve the EDLC, carbon-based electrodes have played an important role. Several nanostructured carbons, including graphene, carbon nanotubes, carbon nanofibers, have been studied, but activated carbon is preferred due to its low cost for production and environmental reasons [15]. Activated carbon can be prepared through physical/chemical processes with baobab fruit shell, coir pith, cucumis melo fruit peel, teakwood sawdust, and it is possible to fabricate electrode of energy storage devices with low cost [16-19]. Nevertheless, AC has been limited to the absorption/desorption process due to its complex pore structure. Several methods have been reported to overcome this problem by developing hybrid materials. [20, 21].

MOFs are porous materials for redox reaction and have a crystal structure by metal ions and organic linker $[14,22]$. MOFs are a promising candidate for SCs due to their large surface area and adjustable pore size [23, 24]. Various studies have been denoted to improve the stability and specific capacitance of MOF materials in the 
application of supercapacitor [25-27]. However, MOFs have two disadvantages: low electrical conductivity and poor chemical/physical stability [28]. Many researchers have attempted to increase the electrochemical properties by combining SIFSIX-3-Ni with carbon materials [29]. For example, CNF/SIFSIX-3-Ni composites as asymmetric supercapacitors exhibit a high specific capacitance $164.5 \mathrm{~F} \mathrm{~g}^{-1}$ [13]. Another effective way is to reduce the particle size of the MOFs material in nanometers that can reduce the ion diffusion distance of the electrolyte. Liu reported the fabrication of hierarchical porous nickelbased SIFSIX-3-Ni, and the supercapacitor has high real specific capacitance $87 \mathrm{~F} \mathrm{~g}^{-1}$ [30].

Electrolyte affects the core characteristics of supercapacitors, and not only determine the driving voltage range but also includes output, internal resistance, charge/ discharge velocity, driving temperature, cycle stability, self-discharge, and toxicity [31]. Generally, carbon electrodes, separators, and liquid electrolytes are introduced and conducted to prepare a conventional capacitor. These mentioned materials for conventional capacitors can be caused by leakage, ignition problems by liquid types of electrolytes. The gel electrolyte can solve problems, such as leakage, ignition, which are problems that occur in liquid electrolytes [32]. In this regard, the stability of gel electrolytes is higher than that of liquid electrolytes [33, 34]. For example, a supercapacitor made of gel polymer electrolytes prepared with a mass ratio of PVA /glycerol/ $\mathrm{NaCl}$ 1: 1: 0.8 and an $\mathrm{AC}$ (activated carbon) electrode has an ionic conductivity value of $62.3 \mathrm{mS} \mathrm{cm}^{-1}$ and a capacitance value of $211 \mathrm{~F} \mathrm{~g}^{-1}$ [34].

In this study, we fabricated a porous structured symmetric supercapacitor by utilizing the large surface area of SIFSIX-3-Ni and AC. For preparation of a solid-state supercapacitor, we used ion-gel electrolyte and $\mathrm{AC} /$ SIFSIX-3-Ni-based electrode was coated by inexpensive doctor blade coating method. The maximum specific capacitance of real supercapacitor was $129 \mathrm{~F} \mathrm{~g}^{-1}$ and a high power density of $1940 \mathrm{~W} \mathrm{~kg}^{-1}$ at a scan rate of $20 \mathrm{mV} \mathrm{s}^{-1}$.

\section{Experimental}

\subsection{Materials}

PVA(Polyvinyl alcohol), EC(Ethylene carbonate), $\mathrm{BMIMBF}_{4}$ (1-Butyl-3-methylimidazolium tetrafluoroborate) were purchased from Sigma Aldrich, and KI was purchased from Junsei Chemical Co,. Ltd to produce solid-state ion gel as an electrolyte. $\mathrm{Ni}\left(\mathrm{NO}_{3}\right) 2 \cdot 6 \mathrm{H}_{2} \mathrm{O}(\geq 97 \%$, nickel(II) nitrate hexahydrate), $\left(\mathrm{NH}_{4}\right)_{2} \mathrm{SiF}_{6}(\geq 98 \%$, ammonium hexafluorosilicate), pyrazine (99\%), and methanol ( $\geq 98 \%$ ) were obtained from Sigma Aldrich to synthesis the SIFSIX-3-Ni. NMP(Nmethyl-2-pyrrolidone), was obtained from Sigma Aldrich, AC(Activated carbon) was purchased from Kansai Coke and Chemicals Co. Ltd, Super-P(Carbon black) was obtained from Imerys Graphite and Carbon, CMC(Carboxymethyl cellulose) was sourced from Tokyo Chemical Industry Co., Ltd, and BM-400B was obtained from ZEON corporation to prepare electrode.

\subsection{Synthesis of SIFSIX-3-Ni metal-organic framework}

SIFSIX-3-Ni was synthesized by the modification of the procedure from the previous report [28]. Briefly, $\mathrm{Ni}\left(\mathrm{NO}_{3}\right)_{2} \cdot 6 \mathrm{H}_{2} \mathrm{O}(0.87 \mathrm{~g}, 3 \mathrm{mmol}),\left(\mathrm{NH}_{4}\right)_{2} \mathrm{SiF}_{6}(0.53 \mathrm{~g}$, $3 \mathrm{mmol})$, and pyrazine $(0.48 \mathrm{~g}, 6 \mathrm{mmol})$ were dissolved in water $(4 \mathrm{~mL})$. The reaction was allowed by heating at $60{ }^{\circ} \mathrm{C}$ for $48 \mathrm{~h}$ under gentle agitation. The blue-color precipitates were collected by simple filtration, and then the acquired powders were rinsed by storing it in $40 \mathrm{~mL}$ of anhydrous methanol for 1 day. Thereafter, the resultant was further purified by a combination of sonication and centrifugation method with rinsing with extra anhydrous methanol. The purification step was repeated 5 times. The obtained resultants were dried at $120^{\circ} \mathrm{C}$ for $12 \mathrm{~h}$ under reduced pressure.

\subsection{Preparation of ion-gel electrolyte}

To prepare ion-gel electrolytes, PVA (1.8 g) was dissolved in distilled water at $90{ }^{\circ} \mathrm{C}$ for $2 \mathrm{~h}$. Then, KI $(3.78 \mathrm{~g})$ was slowly added into the PVA solution and mixed for an hour.
Table 1 Electrochemical properties of the AC/SIFSIX3-Ni composite-based supercapacitor

\begin{tabular}{lllllc}
\hline Sample & $R_{\mathrm{r}}(\mathrm{Ohm})$ & $R_{\mathrm{I}}(\mathrm{Ohm})$ & $\begin{array}{l}\text { Specific capaci- } \\
\text { tance }(\mathrm{F} / \mathrm{g})\end{array}$ & $\begin{array}{l}\text { Energy density } \\
(\mathrm{W} / \mathrm{kg})\end{array}$ & $\begin{array}{l}\text { Power den- } \\
\text { sity (Wh/ } \\
\mathrm{kg})\end{array}$ \\
\hline AC/SIFSIX-3-Ni-0 & 9.5 & 0.7 & 28 & 8 & 420 \\
AC/SIFSIX-3-Ni-0.5 & 3 & 0.2 & 129 & 40 & 1940 \\
AC/SIFSIX-3-Ni-1.0 & 7 & 0.4 & 107 & 33 & 1618 \\
AC/SIFSIX-3-Ni-1.5 & 8 & 0.5 & 87 & 27 & 1318 \\
\hline
\end{tabular}


After that, EC (6.3 g) was dissolved in PVA/KI solution for an hour. Finally, BMIMBF4 (0.57 g) was mixed until being a homogeneous state to create ion gel as a solid-state electrolyte.

\subsection{Fabrication of AC/SIFSIX-3-Ni composite slurry}

All processes for fabricating AC/SIFSIX-3-Ni composite materials were mixed by Thinky Mixer at $2000 \mathrm{rpm}$. CMC was dispersed in NMP for 5 min. Then, SIFSIX-3-Ni $(0.005 \mathrm{~g})$ was added into the NMP/CMC solution and dispersed for $5 \mathrm{~min}$. After that, Super-P $(0.1625 \mathrm{~g})$ was added into the above solution and stirred for $10 \mathrm{~min}$. Afterwards, AC $(0.995 \mathrm{~g})$ was added into the solution and mixed for 13 min. Finally, BM-400B ( $0.125 \mathrm{~g})$ was added to the solution and dispersed for $5 \mathrm{~min}$. The as-prepared sample was denoted as AC/SIFSIX-3-Ni-X, and X stands for the weight percentage of SIFSIX-3-Ni. The weight percentage of SIFSIX-3-Ni in the composite was $0,0.5,1.0$, and 1.5 , and each sample was named as AC/SIFSIX-3-Ni-0, AC/SIFSIX3-Ni-0.5, AC/SIFSIX-3-Ni-1.0, and AC/SIFSIX-3-Ni-1.5, respectively.

\subsection{Fabrication of AC/SIFSIX-3-Ni electrodes}

To fabricate AC/SIFSIX-3-Ni composite-based electrodes, the aluminum substrate was cut with a length of $5 \mathrm{~cm}$ and a width of $1 \mathrm{~cm}$. After cutting the substrate, AC/SIFSIX-3-Ni composite slurry was coated onto the aluminum substrate by a doctor blade method with respect to the variation of SIFSIX-3-Ni wt\% in the composite. The AC/SIFSIX-3-Ni composite-based electrodes were dried at $200{ }^{\circ} \mathrm{C}$ in the oven for an hour to remove residual solvent.

\subsection{Preparation of AC/SIFSIX-3-Ni composite-based supercapacitor}

The AC/SIFSIX-3-Ni composite-based supercapacitor was fabricated by assembling the AC/SIFSIX-3-Ni composite electrodes and ion-gel as a solid-state electrolyte in a sandwich conformation. The ion-gel electrolyte was heated to $40{ }^{\circ} \mathrm{C}$ to activate ion in the solution, and then the fabricated AC/SIFSIX-3-Ni composite electrodes were soaked in the electrolyte solution for $10 \mathrm{~min}$ to diffuse electrolyte into the AC/SIFSIX-3-Ni composite. After then, the electrodes were placed into a fume hood for $30 \mathrm{~min}$ to remove the moisture. Two such AC/SIFSIX-3-Ni composite electrodes with iongel electrolytes were face to face in a sandwich configuration. The prepared full device as a supercapacitor was dried at room temperature for an hour to remove residual solvent and moisture.

\section{Characterization}

\subsection{XRD}

Powder X-ray diffraction (PXRD) was carried out at room temperature on a D/Max 2500/PC (Rigaku) using $\mathrm{Cu}-\mathrm{K} \alpha$ radiation $(\lambda=1.5418 \AA$ ) $) .2 \theta$ scans between $5^{\circ}$ and $50^{\circ}$ with a step size of $0.02^{\circ}$ were performed for a duration of $45 \mathrm{~min}$.

\subsection{FT-IR}

Fourier-transform infrared spectroscopy (FT-IR, Perkin) was conducted in the range of $4000-400 \mathrm{~cm}^{-1}$ at RT.

\subsection{SEM}

Scanning electron microscopy (SEM) images of SIFSIX3-Ni, AC, Super-P and electrode were obtained using a XL30-SFEG. The accelerating voltage was $20.0 \mathrm{kV}$ and the emission current $88 \mu \mathrm{A}$.

\subsection{Cyclic voltammetry}

Cyclic voltammetry (CV) measurements were performed at room temperature with a two-electrode system using a ZIVE SP2 electrochemical workstation (ZIVE-LAB). $\mathrm{CV}$ measurements were recorded over the scan rate at $20 \mathrm{mV} \mathrm{s}^{-1}$ with a potential window of -0.5 to $1 \mathrm{~V}$. The specific capacitance $\left(C_{\mathrm{sp}}\right)$ of symmetric supercapacitor was calculated from the CV curves [34].

$C=\frac{\int I d V}{2 \times v \times m \times \Delta V}$

In this equation, $\mathrm{m}$ is the mass of active material, $\mathrm{v}$ is the scan rate, $\Delta V$ represents the high and low potential limit of the CV measurement, and $I$ is the constant current. Energy density and power density were driven by Eq. (2), Eq. (3) [34]. In those equations, $C$ is the specific capacitance, $\mathrm{V}$ represents the difference between a low potential and a high potential window and $v$ is the sweep time.

$E=\frac{1}{2} \mathrm{CV}^{2}$

$P=\frac{E}{v}$

\subsubsection{Electrochemical impedance spectroscopy (EIS)}

Electrochemical impedance spectroscopy (EIS) was used to inquire about the electrochemical behavior at the 
electrode/electrolyte interface. EIS measurements were performed at room temperature using a ZIVE SP2 electrochemical workstation (ZIVE-LAB), where the frequency range spanned $100 \mathrm{kHz}$ to $0.1 \mathrm{~Hz}$ with an amplitude of $1 \mathrm{mV}(\mathrm{rms})$ at open circuit potential.

\subsubsection{Charge and discharge measurement}

Galvanostatic charge/discharge tests of the AC/SIFSIX-3-Ni composite-based supercapacitor were performed using a battery test system (ZIVE-LAB) between 0 and $1 \mathrm{~V}$ with a constant current density as $0.5 \mathrm{~A} \mathrm{~g}^{-1}$. Capacitance retention was measured by 3000 charge/discharge cycle.

\section{Results and discussion}

\subsection{Physical properties of the SIFSIX-3-Ni}

SIFSIX-3-Ni was synthesized by the conventional solution reaction method to apply for the reinforcing additive to the supercapacitor electrode. The acquired SIFSIX-3-Ni resultant was characterized by powder XRD to determine its typical crystal structure in comparison with its crystallographically defined simulation pattern (Fig. 1a). The XRD pattern of SIFSIX-3-Ni showed that a strong peak at $12.7^{\circ}$ is well consistent with its simulated XRD pattern, as well as other typical peaks derived from SIFSIX-3-Ni were also well matched with the simulated one. FT-IR spectrum of SIFSIX-3-Ni also evidenced its successful synthesis (Fig. 1b). The characteristic peaks at ca. $3118 / 3065 \mathrm{~cm}^{-1}$ and ca. $1435 / 1127 / 1070 \mathrm{~cm}^{-1}$ are derived from the $-\mathrm{CH}$ aromatic bonds and $-\mathrm{CN}$ - bonds in the pyrazine ligands. The strong peak, which corresponded to octahedral $\mathrm{SiF}_{6}$, was also observed at ca. 834 and $773 \mathrm{~cm}^{-1}$. As such, those well support that SIFSIX-3-Ni was well synthesized.

The SEM images of SIFSIX-3-Ni and AC/SIFSIX3-Ni-0.5 electrode are shown in Fig. 2. SIFSIX-3-Ni had cube form and united structure (Fig. 2a). Super-P had a particle that was enough to allow electricity to pass evenly to the electrode (Fig. 2b). AC was a porous structure, a resonable feature that could more effectively support the electron transfer process (Fig. 2c). Figure 2d showed that super-p was well dispersed on the surface of the activated carbon.

\subsection{Electrochemical properties of the SIFSIX-3-Ni-based supercapacitor}

Cyclic Voltammetry (CV) was used to investigate electrochemical behavior under different weight percentages of SIFSIX-3-Ni. Figure 3a showed the CV curve of AC/ SIFSIX-3-Ni composite-based supercapacitor at different weight percentages in the potential window of $-0.5 \mathrm{~V} \sim 1 \mathrm{~V}$. The AC/SIFSIX-3-Ni-0.5 composite-based supercapacitor exhibited and quasi-rectangular shape with widest current rather than other weight percentages of SIFSIX-3-Ni. As the weight percentage of SIFSIX-3-Ni was increased to 0.5 $\mathrm{wt} \%$, the active sites of AC might have been occupied by SIFSIX-3-Ni, which led to a higher $C_{\text {sp }}$ value [35]. However, when the weight percentage of the SIFSIX-3-Ni was greater than $0.5 \mathrm{wt} \%$, excess SIFSIX-3-Ni would decrease the electric conductivity, which led to a smaller $C_{\mathrm{sp}}$ value [35]. According to this result, the SIFSIX-3-Ni, which a porous additive material, could enhance electrochemical property by adding to the AC-based supercapacitor and acting as a role to porous additive material in the electrode [25]. In Fig. 3b, the Nyquist plot showed that all of the AC/SIFSIX3-Ni composite-based supercapacitors gave a semicircle at a high-frequency region, followed by a straight line at the low-frequency region. The semicircle represented the electron transfer rate at the interface of the electrode/electrolyte, which is denoted as charge transfer resistance $\left(R_{c t}\right)$ [35]. In comparing the contents of AC/SIFSIX-3-Ni, it was obvious that the $\mathrm{R}_{\mathrm{ct}}$ value for AC/SIFSIX-3-Ni-0.5 compositebased supercapacitor was smaller than that of other contents of SIFSIX-3-Ni-based supercapacitors. The straight slope
Fig. 1 XRD pattern and FT-IR spectrum of SIFSIX-3-Ni
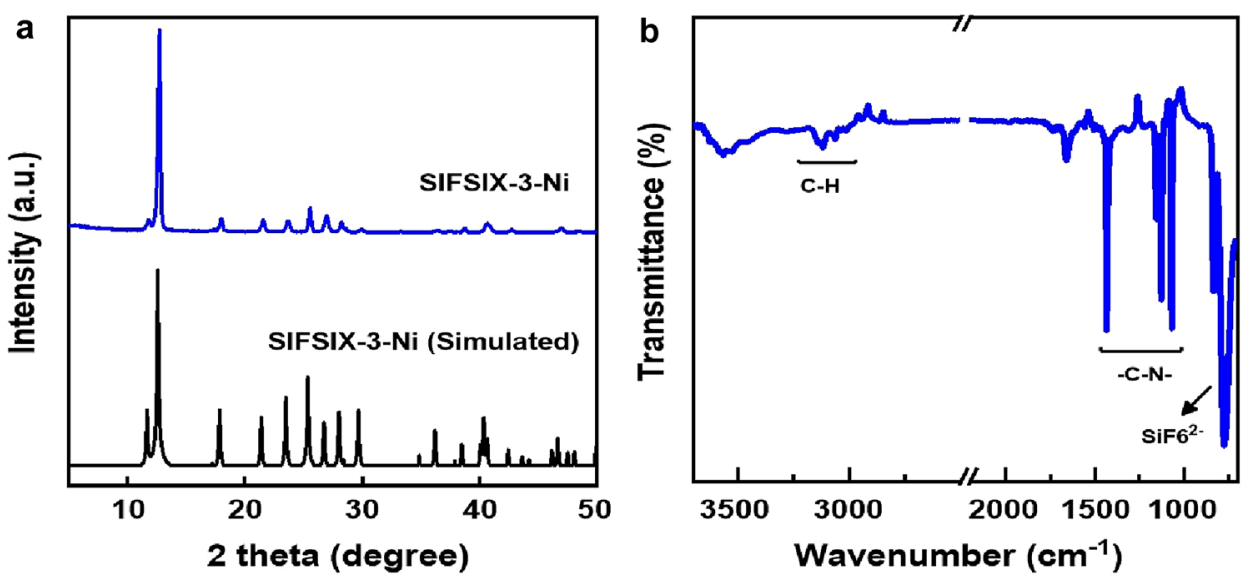
Fig. 2 SEM of a SIFSIX-3-Ni b Super-P c AC d AC/SIFSIX3-Ni-0.5
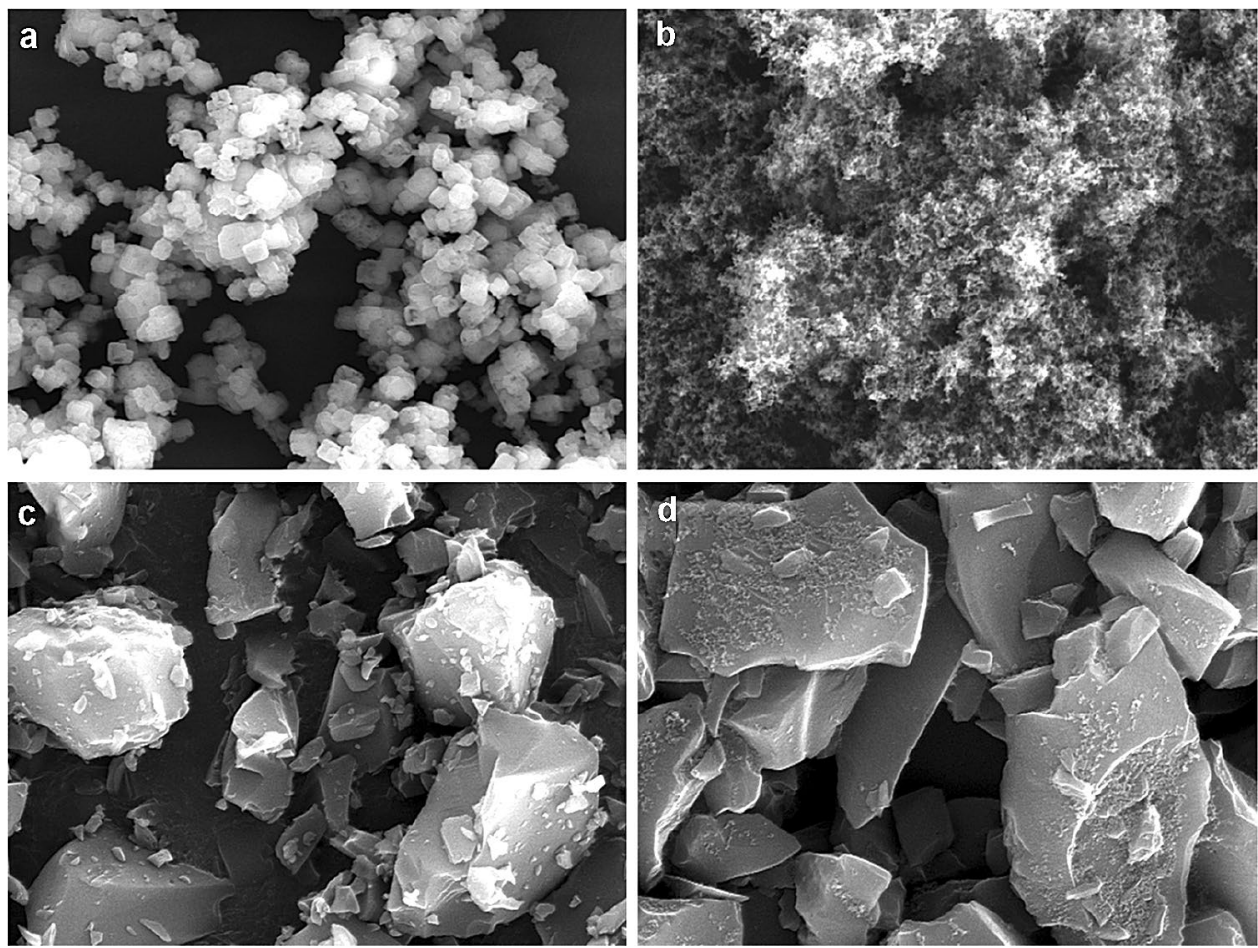

in the low-frequency region represented the ion diffusion from the electrolyte to the electrode surface. The Nyquist plot of AC/SIFSIX-3-Ni-0.5 composite-based supercapacitor showed better electrochemical charge transport properties. The internal resistance value of the AC/SIFSIX-3-0.5 composite-based supercapacitor was $3.0 \Omega$, while the $\mathrm{AC} /$ SIFSIX-3-Ni-1.5 composite-based supercapacitor showed $8.1 \Omega$ (Fig. 3b). The result also explained that the optimized SIFSIX-3-Ni contents could be maintained the electric conductivity and improved electrochemical performance [29]. Figure $3 \mathrm{c}$ showed the charge/discharge curves of $\mathrm{AC} /$ SIFSIX-3-Ni composite-based supercapacitor at $0.5 \mathrm{~A} \mathrm{~g}^{-1}$ of constant current density. The AC/SIFSIX-3-Ni-0.5 composite-based supercapacitor showed also symmetric charge/ discharge graph, the smallest IR drop and the longest discharge time which indicated that the enhanced electrochemical properties of the AC/SIFSIX-3-Ni-0.5 compsite-based supercapacitor. This result also corresponded to CV and EIS data.

The cyclic stability of the AC/SIFSIX-3-Ni- 0.5 composite-based supercapacitor was evaluated by galvanostatic charge/discharge for 3000 cycles. The specific capacitance of the AC/SIFSIX-3-Ni-0.5 composite-based supercapacitor was $98 \mathrm{~F} \mathrm{~g}^{-1}$ after 3000 charge/discharge cycles, which decreased about $20 \%$ from the initial specific capacitance indicating the robustness of the AC/SIFSIX-3-Ni-0.5 composite-based supercapacitor (Fig. 4a). The overall electrochemical performance showed that AC/SIFSIX-3-Ni-0.5 composite-based supercapacitor exhibited a higher specific capacitance (129 $\mathrm{F} \mathrm{g}^{-1}$ ) compared to other contents of the AC/SIFSIX-3-Ni composite-based supercapacitor (Fig. 4b). The remarkable improvement of the electrochemical performance was attributed to the optimized amount of the SIFSIX-3-Ni in the AC/SIFSIX-3-Ni. It is noted that SIFSIX-3-Ni could provide the extra pseudo-capacitance, which also increased the specific capacitance of the AC/ SIFSIX-3-Ni composite-based supercapacitor [36]. The energy and power density were also calculated to evaluate the electrochemical behavior of the AC/SIFSIX-3-Ni composite-based supercapacitor [37]. The AC/SIFSIX-3-Ni-0.5 composite-based supercapacitor showed a high energy density of $40 \mathrm{Wh} \mathrm{kg}^{-1}$ at a power density of $1940 \mathrm{~W} \mathrm{~kg}^{-1}$ (Fig. 4c). The results were much higher than that previously reported systems, including Co2-SIFSIX-3-Ni [2], N, S, and $\mathrm{O}$ tri-doping AC [5], CoNi MOF nanosheet/nanotube [8], $\mathrm{Ni} / \mathrm{SWCNT}$ [13], and NiCo-MOF/acetylene black-based supercapacitors [29]. It suggested that our work is a promising candidate for future energy storage devices and able to scale up to industry. All the analyzed electrochemical preformances data can be found in Table 1.

\section{Conclusion}

The all-solid-state AC/SIFSIX-3-Ni composite-based supercapacitor was successfully assembled, which gave enhanced electrochemical performance by optimizing of the SIFSIX-3-Ni weight percentage in composite. The specific 


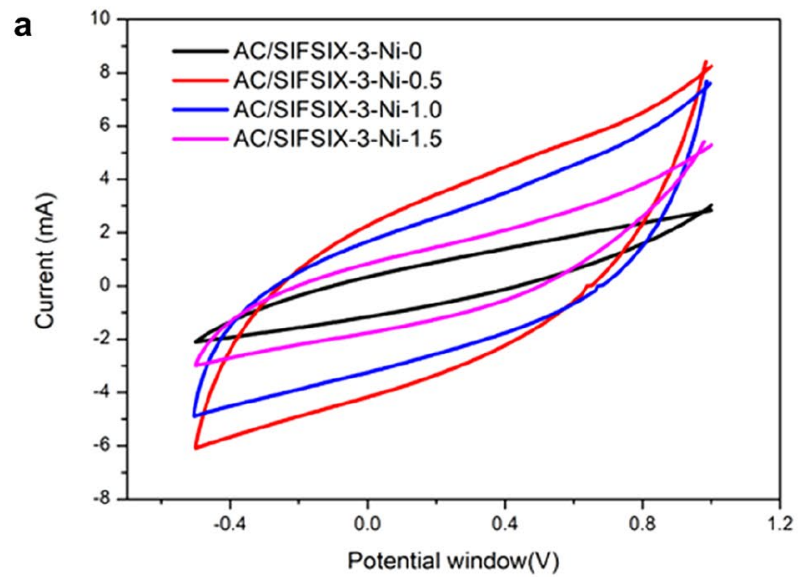

b

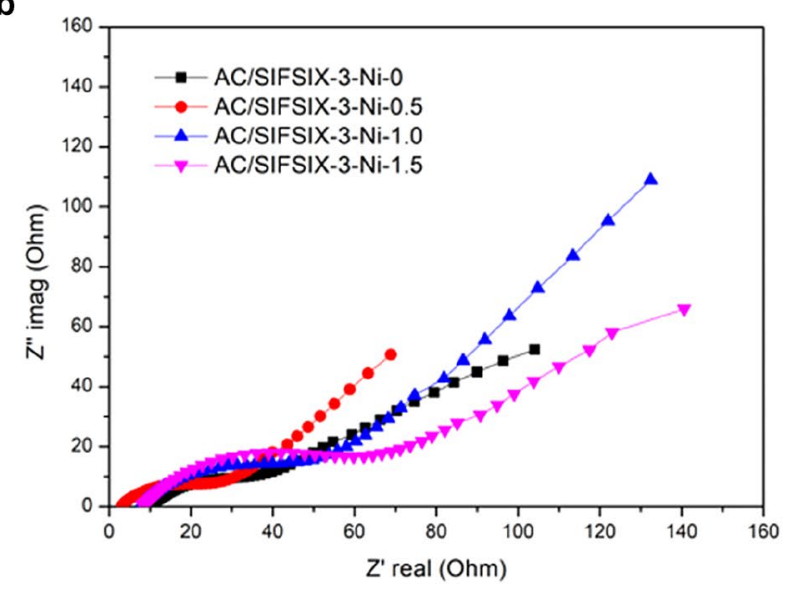

c

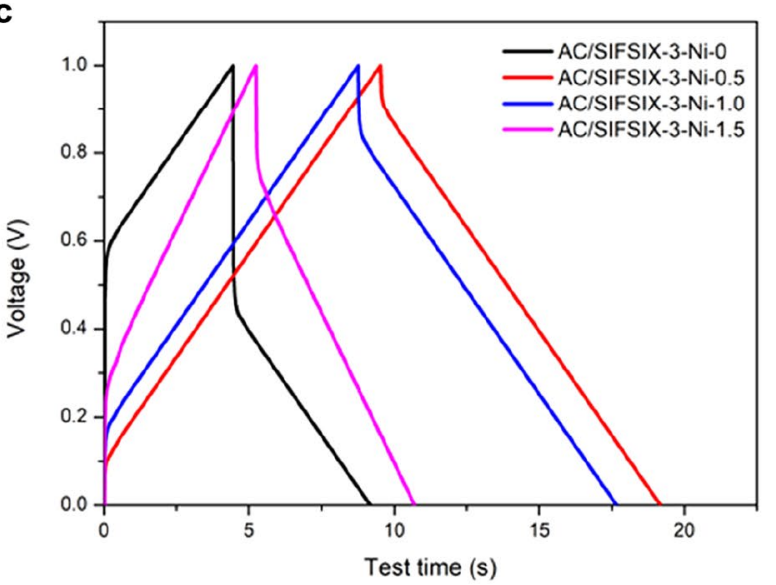

Fig. 3 Electrochemical properties of the AC/SIFSIX-3-Ni compositebased supercapacitor a CV of the AC/SIFSIX-3-Ni supercapacitor under different weight percentage of SIFSIX-3-Ni. b Nyquist plots of the AC/SIFSIX-3-Ni supercapacitor under different weight percentages of $100 \mathrm{kHz}$ to $0.1 \mathrm{~Hz}$ frequency. c Charge/discharge test of the AC/SIFSIX-3-Ni supercapacitor under different weight percentage of SIFSIX-3-Ni with $0.5 \mathrm{~A} \mathrm{~g}^{-1}$ constant current density
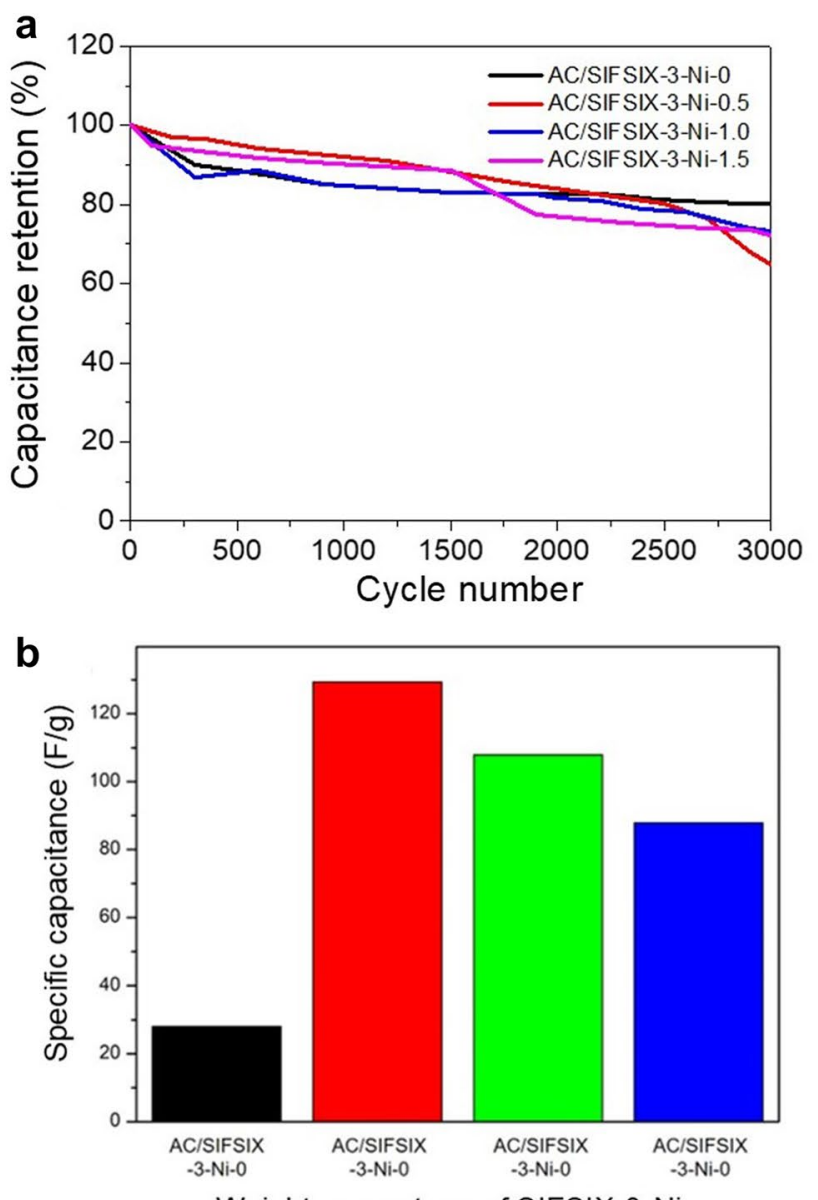

Weight percentage of SIFSIX-3-Ni

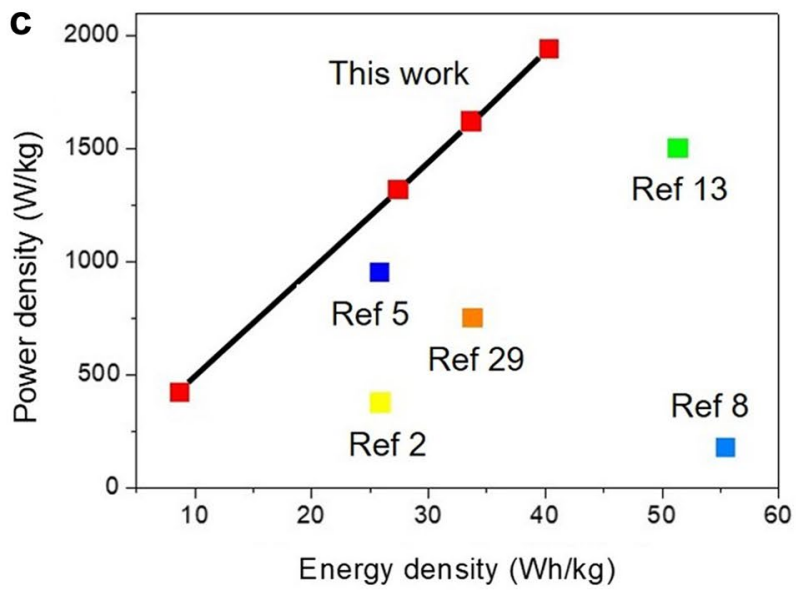

Fig. 4 a Specific capacitance of the AC/SIFSIX-3-Ni supercapacitor under different weight percentages of SIFSIX-3-Ni. b Capacitance retention of the AC/SIFSIX-3-Ni supercapacitor. $\mathbf{c}$ Ragone plots of the AC/SIFSIX-3-Ni supercapacitor under different weight percentages of SIFSIX-3-Ni 
capacitance value of the AC/SIFSIX-3-Ni composite-based supercapacitor showed $129 \mathrm{~F} \mathrm{~g}^{-1}$ at the scan rate $20 \mathrm{mV} \mathrm{s}^{-1}$. The fabricated supercapacitor also maintained $80 \%$ of its initial capacitance value even after 3000 charge/discharge cycles. There are still limitations to carry out optimal electrochemical performances, including capacitance value and energy density of the supercapacitor. To achieve and overcome this issue, electrode materials ionic liquid- and/or organic salt-based electrolytes should be further investigated to improve capacitance and energy density.

Acknowledgements This work was supported by the National Research Foundation of Korea(NRF) grant funded by the Korea government(MSIT) (No. 2019R1F1A105946912).

\section{Compliance with ethical standards}

Conflict of interest The author declare that i have no conflict of interest.

Open Access This article is licensed under a Creative Commons Attribution 4.0 International License, which permits use, sharing, adaptation, distribution and reproduction in any medium or format, as long as you give appropriate credit to the original author(s) and the source, provide a link to the Creative Commons licence, and indicate if changes were made. The images or other third party material in this article are included in the article's Creative Commons licence, unless indicated otherwise in a credit line to the material. If material is not included in the article's Creative Commons licence and your intended use is not permitted by statutory regulation or exceeds the permitted use, you will need to obtain permission directly from the copyright holder. To view a copy of this licence, visit http://creativecommons.org/licenses/by/4.0/.

\section{References}

1. Yue L et al (2019) SIFSIX-3-Ni coating MoS2 structures by hydrothermal intercalation as high-performance electrodes for asymmetric supercapacitors. Chem Eng J 375:121959

2. Wang J et al (2019) Fabrication of 3D Co-doped Ni-based MOF hierarchical micro-flowers as a high-performance electrode material for supercapacitors. Appl Surf Sci 483:1158-1165

3. Marichi RB et al (2017) Efficient, sustainable, and clean energy storage in supercapacitors using biomass-derived carbon materials. In: Martínez LMT, Kharissova OV, Kharisov BI (eds) Handbook of ecomaterials. Springer International Publishing, Cham, pp 1-26

4. Zhao B et al (2017) A high-energy, long cycle-life hybrid supercapacitor based on graphene composite electrodes. Energy Storage Mater 7:32-39

5. Yan J et al (2020) Template-like N, S and O tri-doping activated carbon derived from helianthus pallet as high-performance material for supercapacitors. Diam Relat Mater 102:107693

6. Lee DY et al (2013) Supercapacitive property of metal-organicframeworks with different pore dimensions and morphology. Microporous Mesoporous Mater 171:53-57

7. Li G et al (2019) Construction of hierarchical NiCo2O4@ SIFSIX3-Ni hybrid arrays on carbon cloth as superior battery-type electrodes for flexible solid-state hybrid supercapacitors. ACS Appl Mater Interfaces 11(41):37675-37684
8. Xu S et al (2020) A dual CoNi MOF nanosheet/nanotube assembled on carbon cloth for high performance hybrid supercapacitors. Electrochim Acta 342:136124

9. Wei H et al (2019) Facile synthesis of lotus seedpod-based 3D hollow porous activated carbon/manganese dioxide composite for supercapacitor electrode. J Electroanal Chem 853:113561

10. Zhang X, Xu Y, Ye B (2018) An efficient electrochemical glucose sensor based on porous nickel-based metal organic framework/carbon nanotubes composite (SIFSIX-3-Ni/CNTs). J Alloy Compd 767:651-656

11. Hong J, Park S-J, Kim S (2019) Synthesis and electrochemical characterization of nanostructured $\mathrm{Ni}-\mathrm{Co}-\mathrm{MOF} /$ graphene oxide composites as capacitor electrodes. Electrochim Acta 311:62-71

12. Wang F et al (2019) High-performance non-enzymatic glucose sensor by hierarchical flower-like nickel(II)-based MOF/carbon nanotubes composite. Mater Sci Eng C 96:41-50

13. Tian D et al (2019) Fabrication of two-dimensional metalorganic frameworks on electrospun nanofibers and their derived metal doped carbon nanofibers for an advanced asymmetric supercapacitor with a high energy density. J Power Sources 413:50-58

14. Wu S et al (2019) A review of performance optimization of MOFderived metal oxide as electrode materials for supercapacitors. Int J Energy Res 43(2):697-716

15. Jain D, Kanungo J, Tripathi SK (2019) Performance enhancement approach for supercapacitor by using mango kernels derived activated carbon electrode with p-hydroxyaniline based redox additive electrolyte. Mater Chem Phys 229:66-77

16. Mohammed AA, Chen C, Zhu Z (2019) Low-cost, high-performance supercapacitor based on activated carbon electrode materials derived from baobab fruit shells. J Colloid Interface Sci 538:308-319

17. Mullaivananathan V, Sathish R, Kalaiselvi N (2017) Coir pith derived bio-carbon: demonstration of potential anode behavior in lithium-ion batteries. Electrochim Acta 225:143-150

18. Elaiyappillai E et al (2019) Low cost activated carbon derived from Cucumis melo fruit peel for electrochemical supercapacitor application. Appl Surf Sci 486:527-538

19. Jain A et al (2017) Highly mesoporous carbon from Teak wood sawdust as prospective electrode for the construction of high energy Li-ion capacitors. Electrochim Acta 228:131-138

20. Lee J et al (2017) Asymmetric tin-vanadium redox electrolyte for hybrid energy storage with nanoporous carbon electrodes. Sustain Energy Fuels 1(2):299-307

21. Senthilkumar ST, Selvan RK, Melo JS (2013) Redox additive/ active electrolytes: a novel approach to enhance the performance of supercapacitors. J Mater Chem A 1(40):12386-12394

22. Zhang $\mathrm{H}$ et al (2018) MOF-derived nanohybrids for electrocatalysis and energy storage: current status and perspectives. Chem Commun 54(42):5268-5288

23. Yu C et al (2018) MOF-74 derived porous hybrid metal oxide hollow nanowires for high-performance electrochemical energy storage. J Mater Chem A 6(18):8396-8404

24. Cai M, Loague Q, Morris AJ (2020) Design rules for efficient charge transfer in metal-organic framework films: the pore size effect. J Phys Chem Lett 11(3):702-709

25. Li S et al (2020) Three-dimensional porous carbon/Co3O4 composites derived from graphene/Co-MOF for high performance supercapacitor electrodes. Appl Surf Sci 503:144090

26. Du Y et al (2019) Hollow nickel-cobalt-manganese hydroxide polyhedra via MOF templates for high-performance quasi-solidstate supercapacitor. Chem Eng J 378:122210

27. Zhu C et al (2019) ZnO@MOF@PANI core-shell nanoarrays on carbon cloth for high-performance supercapacitor electrodes. J Energy Chem 35:124-131 
28. Yan Y et al (2016) Facile synthesis of an accordion-like SIFSIX3-Ni superstructure for high-performance flexible supercapacitors. J Mater Chem A 4(48):19078-19085

29. Liu Y et al (2019) Acetylene black enhancing the electrochemical performance of NiCo-MOF nanosheets for supercapacitor electrodes. Appl Surf Sci 492:455-463

30. Du P et al (2018) Fabrication of hierarchical porous nickel based metal-organic framework (SIFSIX-3-Ni) constructed with nanosheets as novel pseudo-capacitive material for asymmetric supercapacitor. J Colloid Interface Sci 518:57-68

31. Pandey GP, Rastogi AC (2012) Solid-state supercapacitors based on pulse polymerized poly(3,4-ethylenedioxythiophene) electrodes and ionic liquid gel polymer electrolyte. J Electrochem Soc 159(10):A1664-A1671

32. Alexandre SA et al (2019) A highly adhesive PIL/IL gel polymer electrolyte for use in flexible solid state supercapacitors. Electrochim Acta 299:789-799

33. Railanmaa A et al (2019) Highly flexible and non-toxic natural polymer gel electrolyte for printed supercapacitors for IoT. Appl Phys A 125(3):168
34. Peng S et al (2019) High-performance and flexible solid-state supercapacitors based on high toughness and thermoplastic poly(vinyl alcohol)/NaCl/glycerol supramolecular gel polymer electrolyte. Electrochim Acta 324:134874

35. Ramli NIT et al (2016) Physicochemical and electrochemical properties of carbon nanotube/graphite nanofiber hybrid nanocomposites for supercapacitor. J Power Sources 328:195-202

36. Raj CJ et al (2018) Polypyrrole thin film on electrochemically modified graphite surface for mechanically stable and highperformance supercapacitor electrodes. Electrochim Acta 283:1543-1550

37. Lv Y et al (2018) Carboxymethylcellulose ammonium-derived nitrogen-doped carbon fiber/molybdenum disulfide hybrids for high-performance supercapacitor electrodes. RSC Adv 8(51):28944-28952

Publisher's Note Springer Nature remains neutral with regard to jurisdictional claims in published maps and institutional affiliations. 